\title{
Hyperbolic-Secant Pulse Propagation in a Single Mode Optical Fiber System
}

\author{
S. H. Lawan \\ Department of Electrical \\ Engineering \\ Bayero University, Kano \\ Nigeria
}

\author{
D. S. Shu'aibu \\ Department of Electrical \\ Engineering \\ Bayero University, Kano \\ Nigeria
}

\author{
S. A. Babale \\ Department of Electrical \\ Engineering \\ Bayero University, Kano \\ Nigeria
}

\begin{abstract}
Results of Hyperbolic-Secant pulse propagation in a singlemode optical fiber communication system are presented. Dispersion results in pulse broadening which limits the information carrying capacity of the fiber. Hyperbolic-Secant pulse propagation model is obtained using split-step Fourier Method from nonlinear Schrodinger Equation. It was found that hyperbolic-secant pulse has a much more uniform pulse broadening and also experience less pulse broadening when compared to a Gaussian pulse at same propagation distance.
\end{abstract}

Keywords: Hyperbolic-Secant, single-mode fiber, dispersion, broadening

\section{INTRODUCTION}

The demand for data communication is growing rapidly due to the increasing popularity of the internet. Ultra-wideband transmission media is needed in order to provide high-speed communications for a much larger number of users. Optical fiber based communication is the excellent alternative to provide high-speed communication [1,2]. Optical fibers as a communication medium for transmission networks started at 1980. Because of the low-loss and wide-bandwidth transmission characteristics of optical fibers, which is one of their foremost advantages over copper cables, have an important role in today's world of communication. As the bandwidth requirement is increasing rapidly, fiber will continue to play its long term pivotal role in telecommunications. Due to its high importance, many industries have been emerged carrying the research on different aspects of the fiber. They are ideally suited for many applications including instantaneous data and movies transmission, high speed internet, medical images, cable, defense, engineering industries etc [3-5]. Fibers that are used for optical communications are wave-guides made of transparent dielectrics whose function is to guide visible and infrared light over long distances, [5]. Signal loss and system bandwidth describe the amount of data transmitted over a specified length of fiber. Many optical fiber properties increase signal loss and reduce system bandwidth [5]. An optical signal is distorted by attenuation and dispersion as it propagates down the fiber. Dispersion can sometime be compensated or eliminated using an excellent design, but attenuation simply leads to a loss of signal, [5]. Finally the energy in the signal becomes deteriorated to the extent that the receiver may not correctly distinguish the signal with sufficient reliability, this is a very serious problem in the system and thereby causing an error to occur. Attenuation therefore determines the maximum distance that optical links can be operated without amplification. For longer distance optical amplifiers are needed. In general, when a system with a very high bandwidth will be used over a long distance, a single-mode fiber is used [5]. Fiber attenuation occurs due to absorption, scattering and bending loses [6]. Dispersion occurs due to wavelength dependence of refractive index while nonlinearities take place due to intensity dependence of the refractive index [6]. There are different types of fiber dispersion and most of them can be reduced significantly using single mode fiber (SMF) rather than multimode fiber, however group velocity dispersion (GVD) is still the limiting factor even in SMF [3, 6-9]. Group velocity dispersion basically broadens the propagating pulse which may cause intersysmbol interference eventually leading to communication errors $[3,5,6]$. It is interesting to understand and be able to evaluate the effects causing these signal degradations such as pulse broadening and fiber attenuation, so as to eventually manage these problems in optical communication systems. As broadening depends on the shape of the pulse, therefore, it is important to investigate the broadening factors of different optical pulses to have minimum pulse degradation [6]. In order to analyze the pulse broadening due to dispersion alone, fiber nonlinearities can be ignored, which means working in the dispersion dominant regime [6]. The model used is split-step Fourier and it was developed by using nonlinear Schrodinger equation that describes the evolution of pulse behavior through SMF [6, 10, $11]$.

\section{METHODOLOGY}

The pulse envelope in time $\mathrm{t}$ at the spatial position $z$, propagating from transmitting to the receiving end of an optical fiber communication system is $A(z, t)$. Pulse propagation in an optical fiber communication system is described by the non-linear Schrödinger equation (NLSE) [6].

$$
\frac{\partial A}{\partial z}+\frac{\beta_{1} \partial A}{\partial t}-\frac{i}{2} \frac{\beta_{2} \partial^{2} A}{\partial t^{2}}+\frac{\alpha}{2} A=-i \alpha_{o}|A|^{2} A
$$

Where

$A \quad$ is the pulse envelope in spatial position $z$ and in time $t$. 
$\beta_{1} \quad$ in the first order dispersion parameter causes pulse delay due to polarization mode dispersion.

$\beta_{2} \quad$ is the second order dispersion parameter causes pulse broadening due to chromatic dispersion.

$\alpha \quad$ is the attenuation coefficient of the fiber

$\alpha_{o} \quad$ is the non-linearity coefficient of the fiber and it is a function of the light strength and

$i \quad$ complex vector notation

The solution of Equation (1) is obtainable using split-step Fourier method. Considering the effect of dispersion only the solution of Equation (1) yields an equation representing the effects of chromatic dispersion (CD) on the input pulse.

$$
A(L, \omega)=\exp \left[\left(\frac{i \beta_{2}}{2} \omega^{2}\right) L\right] A(0, \omega)
$$

It is clear from the above equation that $\mathrm{CD}$ affects the phase of the signal spectrum without changing the spectral power distribution and thereby causing the propagated pulse to be broadened.

$\beta_{2}$ in called the group velocity dispersion (GVD) or simply dispersion, the time delay between two different spectral components separated by a certain frequency interval is determined using the dispersion coefficient $D$ in ps/nm-km, given by [6]

$$
\begin{aligned}
& D=-\frac{2 \pi c}{\lambda^{2}} \beta_{2} \\
& \text { or } \beta_{2}=-\frac{\lambda^{2}}{2 \pi c} D
\end{aligned}
$$

Where $c$ is the speed of light and $\lambda=2 \pi c / \omega$ is the carrier wavelength. The propagation distance after which a Gaussian pulse is broadened by $40 \%$ is termed the dispersion length and is given by [6]

$$
L_{D}=-\frac{t_{o}^{2}}{\left|\beta_{2}\right|}
$$

where $t_{o}$ is the pulse full-width at half-maximum (FWHM) The inputs are represented by a Hyperbolic-Secant pulse Equation (6) and a simple Gaussian pulse Equation (7) and are defined as [6]

$$
A(0, t)=\operatorname{sech}\left(\frac{t}{t_{o}}\right) \exp \left(-\frac{i C}{2}\left(\frac{t}{t_{o}}\right)^{2}\right)
$$

Where $C$ is the chirp parameter

$$
A(0, t)=\exp \left(-\frac{1}{2}\left(\frac{t}{t_{o}}\right)^{2}\right)
$$

Although pulses emitted from many lasers can be approximated by a Gaussian shape, it is very essential to consider other pulse shapes. Of particular interest is the Hyperbolic-Secant pulse shape that occurs naturally in the context of optical solitons and pulses emitted from some mode-locked lasers. The chirp parameter $C$ controls the initial chirp, for the case of unchirped pulses the parameter $C$ equals zero. The results of the pulses are obtained as a sort of comparison between Hyperbolic-Secant pulse and Gaussian pulse.

\section{RESULTS AND DISCUSSION}

Hyperbolic-Secant and Gaussian pulse were taken as input signals and the effect of pulse broadening due to dispersion are studied. The input pulses are shown in Figure 1. Figure 2 and Figure 3 show the dispersed Hyperbolic-Secant pulse and dispersed Gaussian pulse after propagating through SMF respectively. Figure 4 compares the broadening effect on the two pulses and lastly the graphs of pulse broadening versus propagation length are drawn for ease of analysis see Figure 5 .

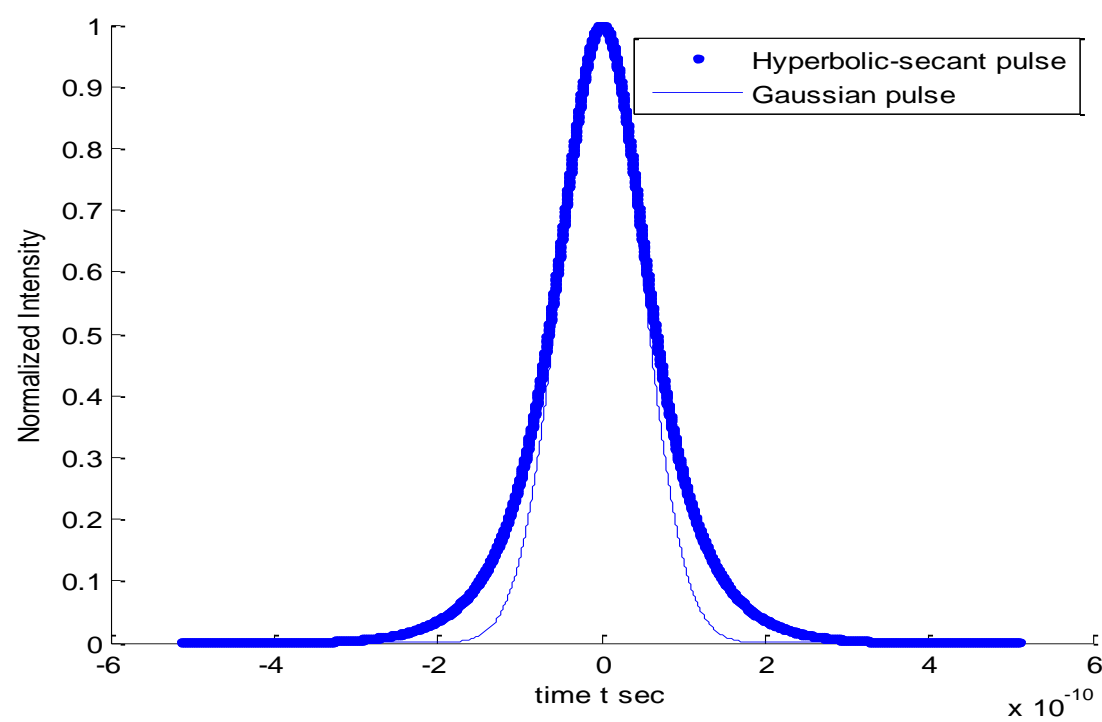

Figure 1: Input pulse (Unchirped Hyperbolic-Secant and Gaussian pulse)

The two pulses shown in Figure 1 both have similar distributions, with Hyperbolic-Secant pulse having stronger wings. 


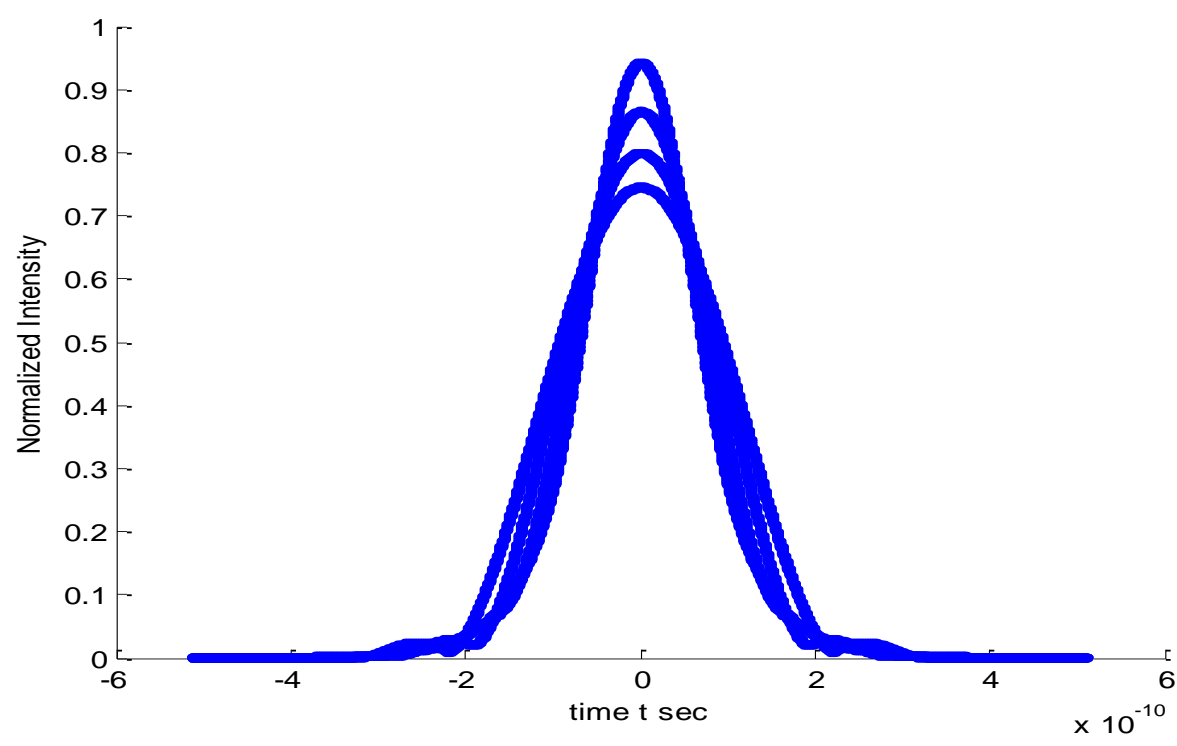

Figure 2: Dispersed Hyperbolic-Secant pulse

Figure 2 depicts Hyperbolic-Secant pulse showing dispersion effects after propagating through a positive dispersion fiber, results taken at 50,100, 150 and $200 \mathrm{~km}$ distances.

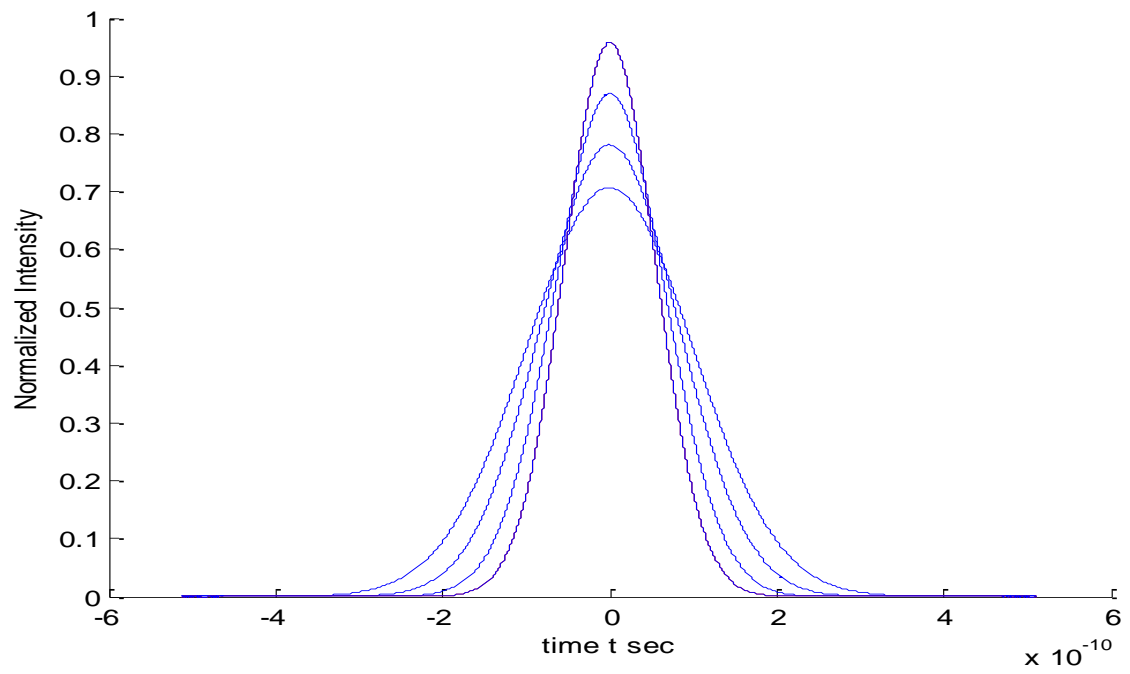

Figure 3: Dispersed Gaussian pulse

Figure 3 presents Gaussian pulse showing dispersion effects after propagating through a positive dispersion fiber, results taken at 50, 100,150 and $200 \mathrm{~km}$ distances. 


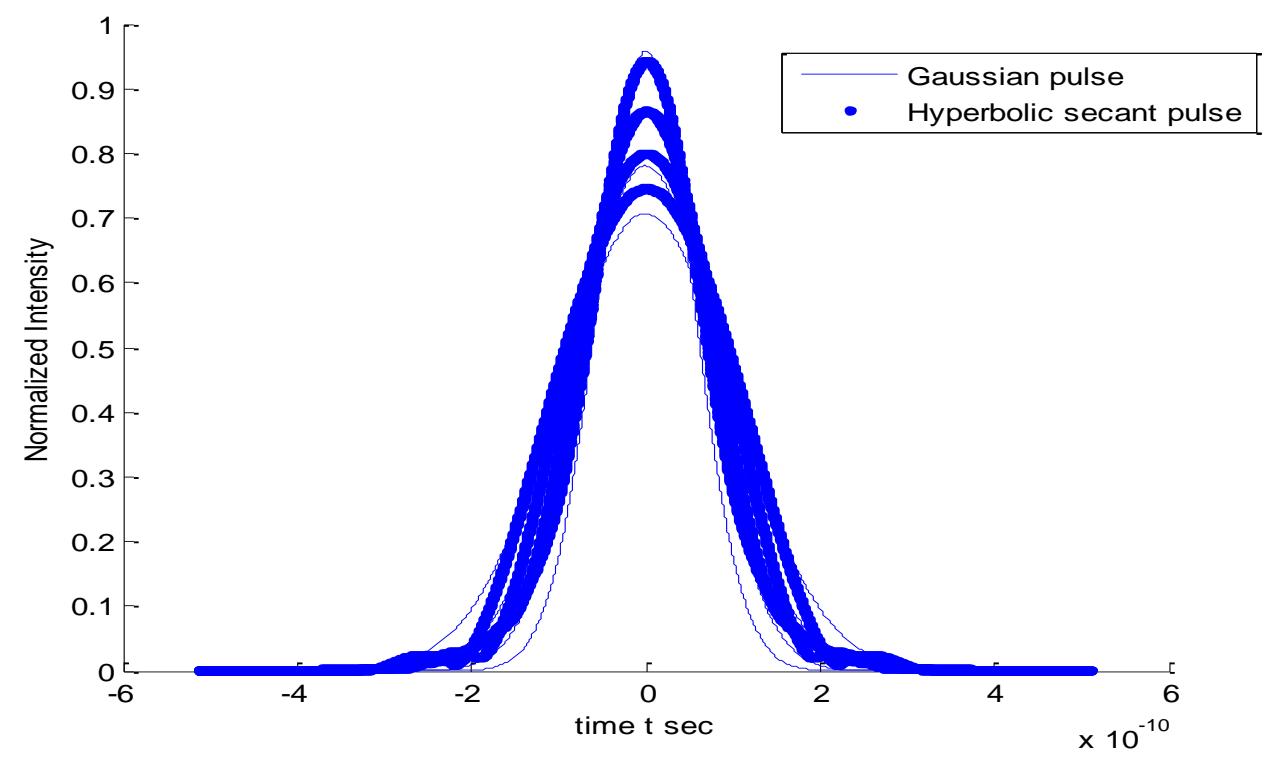

Figure 4: Dispersed Hyperbolic-Secant and Gaussian pulse

Figure 4 shows Hyperbolic-Secant pulse and Gaussian pulse as a way of comparison showing dispersion effects after propagating through a positive dispersion fiber, results taken at 50,100, 150 and $200 \mathrm{~km}$ distances.

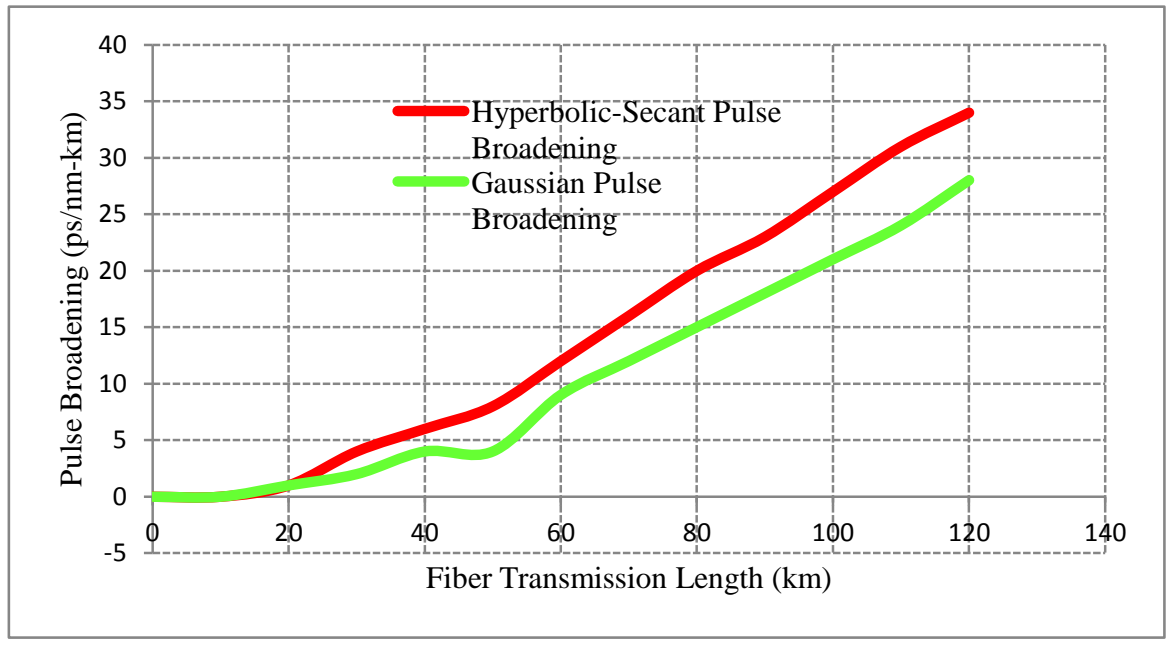

Figure 5: Graph of pulse broadening in ps/nm-km versus dispersion length in km for Hyperbolic-Secant and Gaussian pulse

A comparison shows that the qualitative features of dispersion-induced pulse broadening are nearly indistinguishable for both Hyperbolic-Secant and Gaussian pulses (see Figure 4 and 5). The initial pulse width plays a significant role in determining the pulse broadening, this is in agreement to [6] and that Hyperbolic-Secant pulses have a resistance to broadening and also their broadening is more uniform compared to that of Gaussian pulses at the expense of some nonlinearities in longer transmission distances.

\section{CONCLUSION}

This paper presents numerical simulation of HyperbolicSecant pulse propagation in a single-mode optical fiber communication system. The pulse broadening increases with increase in the transmission distance along the fiber optic link.
It shows that Hyperbolic-Secant pulse has much more uniform pulse broadening when compared to a simple Gaussian pulse. This work can be extended to study and evaluate dispersion by controlling the linewidth of the light source.

\section{REFERENCES}

[1] G. P. Agrawal, Fiber-optic Communication Systems. New York: John Wiley \& Sons. Inc., 2002.

[2] A. Rostami, S. Makouei, and F. Janabi-Sharifi, "Zerodispersion wavelength and mode field diameter managements of ZDSF by optimization technique," International Symposium in Optomechatronic Technologies (ISOT), 2010, pp. 1-4.

[3] N. Sarwar, M. Y. Hamza, and S. Tariq, "Cosh-Gaussian pulse propagation in dispersion dominant regime of 
single mode fiber," Third International Conference on Electrical Engineering, ICEE", 2009, pp. 1-4.

[4] H. J. R. Dutton and I. B. M. Corporation, Understanding optical communications: Prentice Hall PTR, 1998.

[5] S. H. S. Al-Bazzaz, "Simulation of Single mode fiber optics and optical communication Components Using VC++," International Journal of Computer Science and Network Security, vol. 8, pp. 300-308, 2008.

[6] G. P. Agrawal, Nonlinear Fiber Optics, 3rd ed. UK: Academic Press. A Harcourt Sci. and Tec. Co., 2001.

[7] S. Marschall, T. Klein, W. Wieser, B. R. Biedermann, K. Hsu, K. P. Hansen, B. Sumpf, K. H. Hasler, G. Erbert, and O. B. Jensen, "Fourier domain mode-locked swept source at $1050 \mathrm{~nm}$ based on a tapered amplifier," Optics Express, vol. 18, pp. 15820-15831, 2010.

[8] G. Ivanovs, V. Bobrovs, O. Ozoliņš and J. Poriņš, "Realization of HDWDM transmission system,"
International Journal of Physical Sciences, vol. 5, pp. 452-458, 2010.

[9] M. Aliramezani and S. M. Nejad, "Numerical analysis and optimization of a dual-concentric-core photonic crystal fiber for broadband dispersion compensation," Optics \& Laser Technology, vol. 42, pp. 1209-1217, 2010.

[10] O. V. Sinkin, R. Holzlöhner, J. Zweck, and C. R. Menyuk, "Optimization of the split-step Fourier method in modeling optical-fiber communications systems," Journal of Lightwave Technology, vol. 21, p. 61, 2003.

[11] G. Muslu and H. Erbay, "Higher-order split-step Fourier schemes for the generalized nonlinear Schrödinger equation," Mathematics and Computers in Simulation, vol. 67, pp. 581-595, 2005. 